\title{
An active connection mechanism for modular self-reconfigurable robotic systems based on physical latching
}

\author{
A. Sproewitz, M. Asadpour, Y. Bourquin and A. J. Ijspeert
}

\begin{abstract}
This article presents a robust and heavy duty physical latching connection mechanism, which can be actuated with DC motors to actively connect and disconnect modular robot units. The special requirements include a lightweight and simple construction providing an active, strong, hermaphrodite, completely retractable connection mechanism with a 90 degree symmetry ${ }^{1}$ and a no-energy consumption in the locked state. The mechanism volume is kept small to fit multiple copies into a single modular robot unit and to be used on as many faces of the robot unit as possible. This way several different lattice like modular robot structures are possible. The large selection for dock-able connection positions will likely simplify self-reconfiguration strategies. Tests with the implemented mechanism demonstrate its applicative potential for self-reconfiguring modular robots.
\end{abstract}

\section{INTRODUCTION}

Modular self-reconfigurable (MSR) robotic systems are characterized as being composed of multiple robotic units, each unit having few degrees of freedom (dofs) and a high degree of autonomy (energy- and computational autonomy, an own sensor system and communication capabilities).

A single MSR unit cannot usually do much on its own, but by combining several units into a larger structure various tasks can be performed.

The number of different types of MSR units in the MSR system is very low, typically one ([1], [2], [3], [4], [5]) or two active units [6] are used, or a combination of one active and passive unit type ([7], [8]). These main characteristics distinguish MSR robotic systems from monolithic robot structures (e.g. humanoid robotic systems) and create specific advantages and disadvantages when comparing both of them.

\section{A. Advantages of MSR}

Common reasons for developing MSR robotic systems are the a) generation of redundant systems. Because the overall robot structure is made from a lot of identical units, one failing module can be easily replaced. In the best case this is done autonomously, resulting in a self-repairing system. b) If needed, a MSR system assembled into e.g. a quadrupedshaped robot can alter itself into a snake-shaped system, crawling through a hole non-accessible by the quadruped configuration [9]. Similar strategies are used in substrateMSR robotic systems [10]. For transport MSR systems can self-reconfigure into a small-sized and dense cube. Being

Alexander Sproewitz, Masoud Asadpour, Yvan Bourquin and Auke Jan Ijspeert are with the School of Computer and Communication Sciences at EPFL, Swiss Federal Institute of Technology, CH 1015 Lausanne, Switzerland. Email: (alexander.sproewitz, yvan.bourquin, auke.ijspeert)@epfl.ch, masoud.asadpour@ieee.org

${ }^{1}$ commonly also used: 4-way symmetry

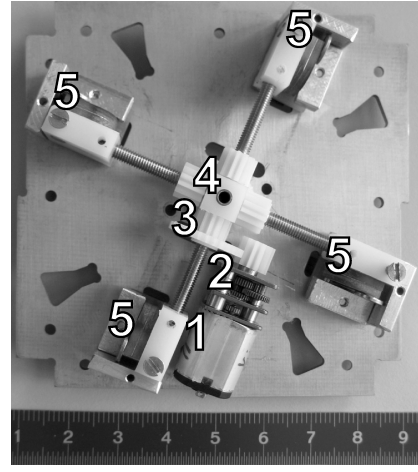

Fig. 1. Top view on a prototype of the active connection mechanism, shown is one connection plate, the DC motor (1) with gearbox (2), a spur gear system (3), the distributing gearbox (4) and the latch mechanism (5).

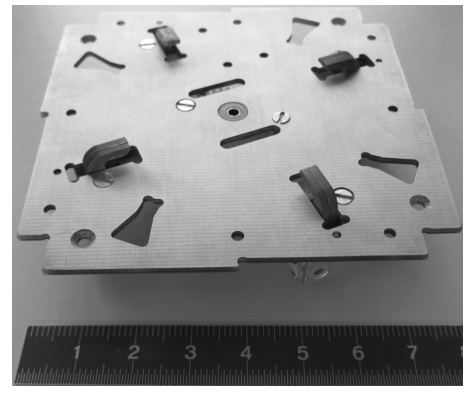

Fig. 2. Bottom view. Scale of the ruler in $[\mathrm{cm}]$, the shown board size is $8 \mathrm{~cm}$ by $8 \mathrm{~cm}$. The shown board, but without the actuation (DC motor) and with two (diagonally) oriented latches being replaced by spring loaded blockers is used for the load experiments described in section III-A.

able to experiment with different robot configurations using only a limited set of units makes MSR and modular robotic (MR) systems also a c) very attractive tool for scientific research [11]. Another application is within d) swarm robot applications [12]. By dividing into several smaller units, area exploration can be performed in parallel. Once an optimal route is found, the MSR structure is re-assembled and can follow the optimal path. Often mentioned is e) an advantage in price for MSR robotic systems. If many of them are being produced costs can be reduced. However, this argument is mainly valid for a mass-market product. Projects like the open Molecubes Project [13] could support the development of low-cost MR systems.

To sum it up, MSR robotic systems are specially suited in areas where a high degree of autonomy is needed, such as hazardous areas, disaster sites or in places for which it is too expensive to send human workers (space or deep sea 
applications).

\section{B. Disadvantages of MSR}

Arguments against the usage of MSR robotic systems are: because a MSR system is made from identical units, it is imperatively featuring A) less optimal dynamic and geometric properties when comparing it to a monolithic structure optimized for a specific task. MSR systems usually feature B) many active and complex units, hence the overall system can become costly. A further challenge is the C) coordination of a high amount of units for e.g. locomotion tasks. Avoiding internal collision and creating the right connection for selfreconfiguration is only possible if $\mathrm{D}$ ) efficient reconfiguration strategies are available.

\section{Hardware of $M S R$}

A big part of the feasibility of a MSR system derives from a highly robust, cheap, well balanced mechanical and electronic design of the single units. That includes the possibility for high speed communication among the modules, sufficient energy and processing power, a sensor architecture delivering autonomy to the MSR units, and a properly chosen actuation power. Finally the MSR robotic units need to feature a reliable and robust active connection mechanism for interconnecting the MSR units.

This work concentrates on active connection mechanism principles based on physical latching, rather than on connection mechanisms based on electro-static, electro-magnetic, or permanent-magnetic forces. We give a brief discussion for this choice (Section II). We explain the resulting robustness against misaligned connection plates and the background for heavy-duty connectors, together with details for the implementation of our prototype (Section III). Section IV refers briefly to Self-Reconfiguration Planning to show its impact on the latch design. In Section V we give details for the new feature of an active connection mechanism for MSR robotic systems implemented in Webots, a simulation environment we are using. We discuss our approach in Section VI.

\section{Choosing A PRINCIPLE}

Active connection mechanisms (ACMs) can roughly be characterized by the forces they are using to hold MSR robot units together; a) permanent-magnet based magnetic force, e.g. [1], [14] b) electromagnetic forces, e.g. [15], [16] c) electrostatic forces [17] and d) ACMs based on physical latches or pins, e.g. [4], [8], [18], [19], [2], [20], [21], [7], [12], [5]. There is no MSR robotic system known to the authors using e) atmospheric pressure (e.g. suction-power connectors), however hydrostatic pressure can be used to connect modules [21]. Some of the above principles are actually used in combination; Molecubes are using electromagnets that "selectively weaken and strengthen connections" [16], using additional permanent magnets.

\section{A. Demands on an active connection mechanism}

Locking the dof: If no movement between the connection plates is desired, a connection mechanism interconnecting two MSR units has to lock all the open degrees of freedom (dof). Therefore, blocking of $\mathrm{x}, \mathrm{y}$ and $\mathrm{z}$-translation (see also Fig. 4) movement or blocking of rotation around the z-axis and translation in the $\mathrm{z}$-direction are needed. The $\mathrm{z}$-axis is orthogonally oriented towards the connection face, the $y$ and $\mathrm{x}$-axes follow the right hand rule.

Guidance, $x$ and $y$ position: This is needed to bring together two opposing faces in such a way that the actual connection mechanism is able to lock the connection. Several principles are used: a) Passive guidance by structured surfaces (e.g. used in [16], [21]). By using the pins as guidance, together with a wiggling movement of the MSR units, positioning can be achieved as well ${ }^{2}$. However, the MSR unit must be able to perform the required wiggling movement. This requires an appropriate choice of dof. The bigger the pins, latches, grooves and surface structures are, the easier and more robust is the docking procedure ${ }^{3}$. b) Active guidance is achieved by using e.g. IR-sensors [2] or camera systems [9] to guide the searching movement of the ACM. c) When using ACMs based on magnetic or electrostatic forces, the passive selfalignment features by the magnetic flux or the electric field, respectively, can be used.

Grasping movement, z-translation: If two connection faces are aligned in the $\mathrm{x}$ and $\mathrm{y}$ directions, it is advantageous if the ACM is able to overcome gaps in the $\mathrm{z}$-direction by a grasping/scooping movement. These gaps can derive from dust and obstacles between the connection plates, from uneven surfaces (e.g. after usage or from bending under stress), or from a slight angular misalignment of the plates. Magnetic and electrostatic principles automatically include forces in the z-direction, certain physical latches based on hook designs do this as well (see Fig. 3 for hook trajectories). However physical latching systems based on pin \& hole systems do not support this ability. A single MSR unit (unita) must be fixed in position and the approaching unit-b needs to insert the pin into the hole until the latching procedure can lock the mechanism. This can lead to opposing forces between the two MSR units if they are not perfectly aligned. In case unit-a is not sufficiently fixed and the mounting process is not completely aligned, unit-a might simply be pushed away by unit-b. Connection systems based on screws (using rough threads ressembling connectors for cameras with tripods) can be seen as an extreme case of a hooking system-the challenge will most likely be to feed-in the thread. Communication, power supply: Any connection mechanism presents a barrier between the modules, but stable and high-speed communication between the MSR units is crucial. It is also sometimes desirable to have power lines interconnecting the modules (for relayed recharging, in case of power-units...). For communication, some systems use IR-communication or even re-use the IR-sensors. Others rely on wireless communication between the modules [23]. Like this direct connections anywhere inside the robot assembly

\footnotetext{
${ }^{2}$ also "blindly" or by judging from sensor values measuring internal bending [22]

${ }^{3}$ extreme values are achieved with e.g. the Dragon connector [19]
} 
are possible, plus they are not dependent on the quality of the contact connectors. Contact connectors are widely used and can relay information and power. If the interconnecting hooks or pins are reused as contact connectors one can provide perfectly flat surfaces for the MSR units [8]. Standard contact connectors are based on spring contacts will slightly protrude the surface.

Symmetry and gender: When assembling a MSR robot system from several units, a high number of docking orientations is desirable. A 90 degree symmetry of the connectors on the connection faces refers to 4 possible docking positions. If the $\mathrm{ACM}$ is a hermaphrodite, the self-reconfiguration planning can be simplified (see Section IV for details.).

Connector strength, power consumption, geometry: The overall connector must provide shear strength and withstand tensile stress. Structured surfaces can be designed to cope partly or completely with shear forces (e.g. for permanent magnetic connectors being vulnerable to shear forces). Because MSR robotic systems are designed as autonomous systems, they usually have a restricted battery capacity. Therefore, the ACM should consume power only during the docking and decoupling process. This is mostly not valid for electro-magnetic $\mathrm{ACMs}^{4}$. If a simple hook is used and the actuation system is reversible, $F_{x}$ (see Fig. 3) will insert a torque to the hook trying to open the connection. A trivial demand is a flat and small-sized ACM design-the more attaching surfaces are available the more global robot configurations are possible. The desired properties of our new connector include properties of a MSR system similar to [9], [2], with MSR units based on a cubic grid $(110 \mathrm{~mm}$ grid size). Humans will interact with the MSR system (safety issue), and the MSR systems should be able to withstand relatively high forces when combined into a global robot. It should be small sized and should not consume energy in the docked state. Self-alignment in $\mathrm{x}$ - and $\mathrm{y}$ - directions and robustness against $\mathrm{z}$-axis misalignment is preferred. In summary, the aimed features are:

1) Physical latching with no $F_{x}$-fraction (Fig. 3a). The tensile force should possibly remaine inside the hook (as in [8]). An electro-static ACM is not human-friendly (current systems use high voltages directly applied on the surfaces), whereas pure electr-magnetic ACMs consume energy in the holding state. Permanent magnetic force-based systems would feature self-alignment, however the applied forces on the global robot would likely require very strong permanent magnets. To create strong magnetic fields, enough magnets and sufficient flux-conducting material is required and the connector is likely to become heavy.

2) A hermaphrodite connector with 90 degree symmetry. If possible, the latches should serve a dual-purpose for power and communication relay.

\section{DESIGN \& IMPLEMENTATION}

Looking at the above demands and the existing solutions for active connection mechanisms, two MSR robot systems

\footnotetext{
${ }^{4}$ Only if the electro-magnetic ACM is used in parallel to permanent magnetic forces weakening them for disconnection.
}
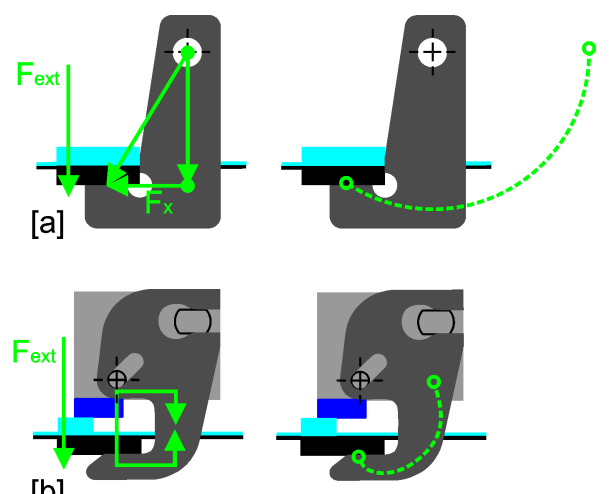

[b]

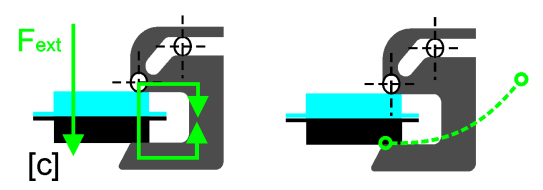

Fig. 3. In the left: applying forces in several hook design principles, on the right trajectories for the corresponding latch design. Walls are like in Fig. 5, $F_{\text {ext }}$ (e.g., $F_{\text {tensile }}$ ) is an external force pulling away the neighboring module. [a] simple hook principle, [b] proposed design, [c] clamping principle as used with AMAS [8] and MTRAN III [9], the actual shape and sizes differ. In design [b] and [c] applying force-lines will be directed only through the latch and the direct hull area, in design [a] force-lines go through the hook, axis and hull structure. The "scooping" motion in design $[b]$ is visible, a property taken from the simple hook design.

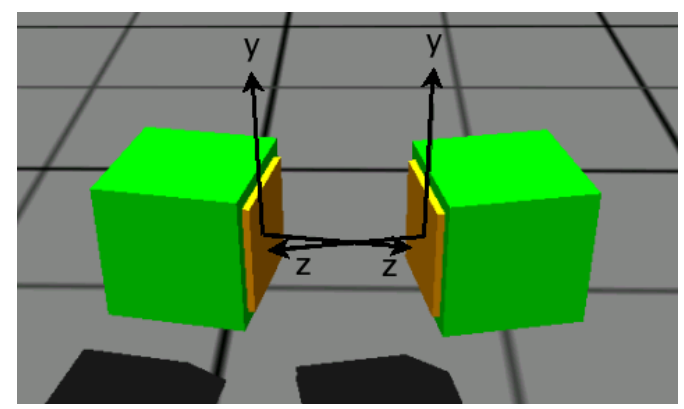

Fig. 4. Connector axes. For the load experiments described in section IIIA two real connectors are assembled, positioned as in the above picture and being connected by two latches, any actuation (DC motor) is removed during load test. $F_{\text {shear }}$ in the load experiments is directed $-y, F_{\text {tensile }}$ along a $z$-axis. $M_{\text {shear }}$ uses the $z$-axis for rotation and a $40 \mathrm{~mm}$ lever (half the cube size).

using a similar latching system remain, satisfying almost all requirements: AMAS [8] and MTRAN III [18]. Because we are planning for a MSR robotic system with relatively high applied forces ${ }^{5}$ and less accuracy for $\mathrm{z}$-alignment ${ }^{6}$, we were searching for a design featuring the grasping movement of a hook (Fig. 3), together with the capability to trap $F_{\text {ext }}$ inside the hook only (Fig. 3 representing-roughly-the AMAS and the MTRAN III latching system).

Therefore, we use a system producing a "scooping" movement (Fig. 6 and Fig. 5a to Fig. 5f). To redirect $F_{\text {ext }}$-lines into the hook, a latch must be shifted between the hook

\footnotetext{
${ }^{5}$ Therefore connection surfaces might get bent by strain, resulting in $\mathrm{z}-$ misplacement.

${ }^{6}$ longer chains for self-reconfiguration movements than MTRAN III
} 

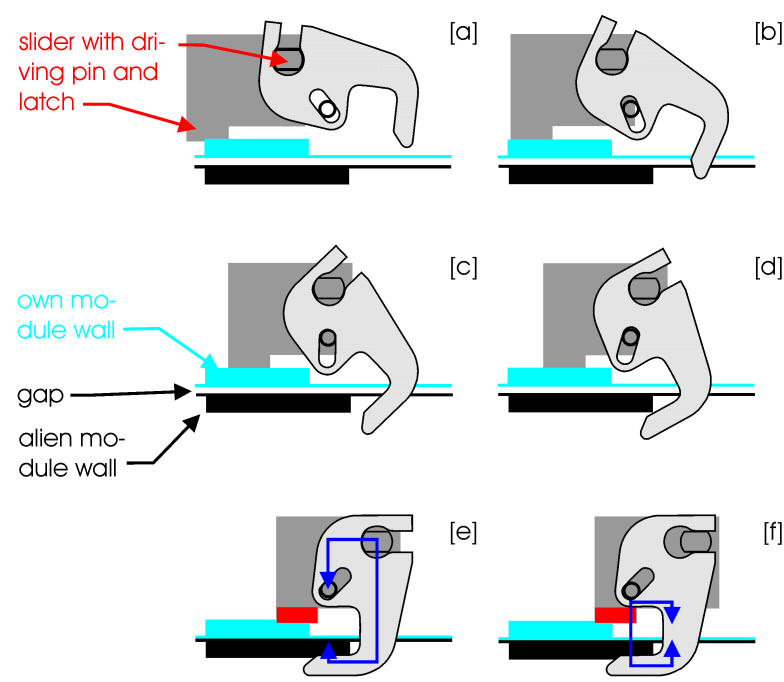

[e]

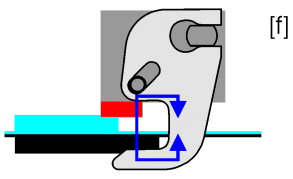

alien module wall pulled close

at the end of the movement

Fig. 5. Latching mechanism in the sectional view. The latch shifting in (Fig. 5e) is closing the force-lines.

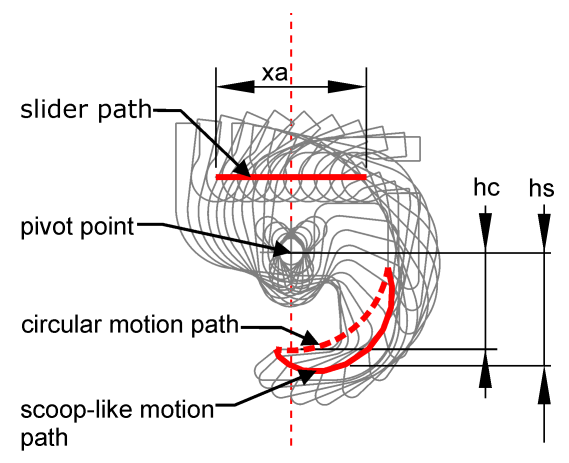

Fig. 6. Steps of $1 \mathrm{~mm}$ of the slider motion are overlaid, the tip of the hook shows a scoop-like motion path (bold continuous line, due to pin-in-slot guidance) other than a circular motion (bold, dashed line) when using a pin-in-hole guidance. $h_{s}-h_{c}$ corresponds approximately to the maximum misplacement $d_{z}$ discussed in section III-A.

and the connection faces. In case $F_{\text {ext }}$ is not too high, the system can be used without the translational latch and the specially shaped driving pin, basically representing a dense standard hook design. The latch is directly attached to a slider moving the leading pin, however the rotation of the hook must be stopped (Fig. 5e). Therefore the driving pin is specially shaped and enables a detachment of the driving pin from its rotating position, after reaching the position shown in (Fig. 5e). The slider continues shifting a latch underneath the hook until positioned (Fig. 5f). For the inverse movement of the slider, the latch is positioned such that the rotation of the hook starts immideately after the latch has left the gap between the wall and the hook. This timing is important and can be supported by a spring holding the hook. The position of the hook might otherwise become undefined before the driving pin reaches its rotation point again.

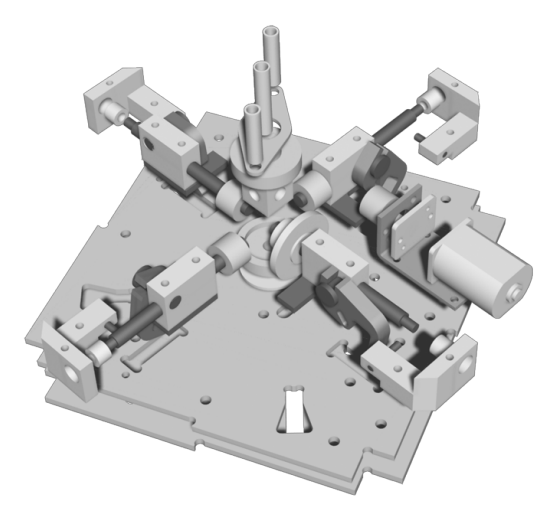

Fig. 7. Exploded view for the latching system with four latches. Screws are not shown, the board size is $80 \mathrm{~mm}$ by $80 \mathrm{~mm}$. We also designed a version with only two latches, what simplifies the torque distribution, lowers the friction of the overall setup and, as the main reason, decreases the board size to a circle of $65 \mathrm{~mm}$ diameter at a height of $16 \mathrm{~mm}$.

\section{A. Load and alignment tests for the latching system}

As we are aiming for a heavy-duty latching mechanism, we tested our design under the following conditions: an "active" connection board (B1) as in Fig. 1 is fixed by screws against a wall. We removed the DC motor (the screw drive is non-reversible) and used only two latches (see Fig. 5 for a latch design). We replaced the other two latches each by a spring-loaded blocker without latching capability to cope for applying torque between the two connectors. Each of the latches is made from two identical pieces and has a thickness of $3.2 \mathrm{~mm}$ (two $1.6 \mathrm{~mm}$-thick glass-fiber reinforced plastic pieces being copper-plated and soldered together) with a cross-section area of approximately $7.7 \mathrm{~mm}^{2}$ at its weakest point.

The opposing connection board (B2) is a blank board with notches only, such that the two latches from board B1 can grab onto it. The two blockers from B1 lock against rotation only. Three different load tests were carried out successfully: $F_{\text {tensile }}, F_{\text {shear }}$ and $M_{\text {shear }}$ (described in Fig. 4), with each using a load of $18 \mathrm{~kg}$ (static load). For $M_{\text {shear }}$ the torque was applied using the housing as a lever (lever length of $40 \mathrm{~mm}$ ), resulting in a torque of $M_{\text {shear }}=7 \mathrm{Nm}$. Using only two latches instead of four simplifies the design, however forces and torque are not applied as evenly as with four latches, and the supporting walls of B1 and B2 will eventually bend.

Our latch design is aiming to compensate for an angular misalignment of the $y$-axis ( $d_{y y}=1.7$ degrees) or a distance misalignment ( $z$-axis) up to $d_{z}=1 \mathrm{~mm}$ purely by the "scooping" movement into the notches of B2. These values are measured using the 3D CAD model in Solidworks and a latch designed to grab a plate of $1.6 \mathrm{~mm}$ thickness. The bigger the latch can be designed, the higher the misalignment is allowed to be. The slope at the tip of the latch should compensate for $1 \mathrm{~mm}$ more $d_{z}$-misalignment.

\section{B. Actuation}

Because we are aiming for a minimal motor torque and an acceptable connection time, the lever for the driving pin and 
the axis of evolution is kept relatively big. We can currently achieve an overall design of $16 \mathrm{~mm}$ height including the actuators. A four-latch and a two latch design fit into a circle of an $80 \mathrm{~mm}$ and $65 \mathrm{~mm}$ diameter, respectively. We have the choice for two low-cost DC motor/gear box combinations: a $12 \mathrm{~mm}$ flat design motor with a $75: 1$ gear ratio $(15 \mathrm{sec}$ to retract four latches) and a $15 \mathrm{~mm}$ flat design motor with a 30:1 gear ratio (5 sec to retract four latches). We are still working on decreasing the friction inside the actuation system (resulting from distributing the torque from one DC motor onto 4 latches, including 4 worm gears). Eventually this will enable us to use smaller gearing ratios and it should lead to faster docking procedures.

\section{SELF-RECONFIGURATION PLANNING}

Several features of the latching design, such as its hermaphrodite properties and the small sized design were chosen to support the self-reconfiguration planning (SRP). The goal of self-reconfiguration planning is to design an optimal algorithm that minimizes the number of steps required to reach a final configuration, starting from an initial configuration. The SRP process must address three essential questions:

1) Possibility: Does any path exists between the initial configuration of MSR units and the final configuration of the MSR robot?

2) Optimality: In cases where multiple paths exist, which of them is optimal in terms of some optimality criteria e.g. number of reconfiguration steps?

3) Computability: How long does it take to find the optimal solution out of the possible solutions? And, how does the computation time scale up with an increase in the number of modules?

In many cases, hermaphrodite connectors are helpful in simplifying the reconfiguration process, but they can raise some problems as well. In male/female connections, attention must be paid to bring male connectors close only to female ones and vice versa. However, in hermaphrodite connections all connection points are compatible. Therefore it is feasable to reach a larger number of configurations from a specific configuration. This means a bigger solution space as well as a bigger search space. A bigger solution space gives a higher chance for finding a feasible reconfiguration path between two configurations (possibility). It thus increases the chance of finding shorter reconfiguration sequences (optimality). A bigger search space, however, results sometimes in a simpler reconfiguration process and sometimes makes it more complicated. If by having hermaphrodite connectors a kind of high-level planning is made possible, the SRP process would not only take a shorter time but would also scale very well to large numbers of modules (computability). On the other hand, if high-level planning is not possible, the SRP process usually applied would be a random search in a big search space. That means a longer computation time would be required.

\section{ACTIVE CONNECTION MECHANISM IN SIMULATION ENVIRONMENT}

In order to validate our design and to be able to make predictions for bigger MSR structures using our latch design, we are collaborating with Cyberbotics Ltd and using their Webots software described as "a rapid prototyping environment for modeling, programming and simulating mobile robots" [24]. In the case of modular robots, the Webots controllerprogram can access simulated sensors and actuators, detect the presence of compatible connectors during run-time and also dynamically connect and disconnect robot units from each other. Because possible connection mechanism principles differ greatly, Webots provides a general framework, that allows for both symmetric and asymmetric mechanisms, any number of rotational docking positions, user-defined docking tolerance, etc. We recently added several new features for the active connection mechanism to get as close as possible to real-world MSR robotic systems. The newly developed "snap" mechanism allows to automatically align connectors when they are close enough and when the latch mechanism is triggered. Two snapping parameters distanceTolerance and rotationTolerance correspond to the above (section III-A) measured parameters $d_{z}$ and $d_{y y}$, respectively. Transferring both values from the latch design to the simulation will hopefully provide a robust simulation basis for active latching. An additional parameter axisTolerance depends on the angle between the two z-axes. When the controller program "locks" the connection mechanism, the simulation automatically aligns the connectors; the two plates are pulled together, and they are rotated to the closest matching 90 degree docking position. Another feature we recently implemented is the "rupture simulation", that allows to simulate the rupture of the connection mechanism under external stress. An example is shown in Fig. 8 where two connected robot units (A, B) are dropped from 2 different heights onto a solid block. The connection breaks when the units are dropped from a higher location (Fig. 8-1 and 8-2) while it does not break when dropped from a lower location (Fig. 8-3 and 8-4). In this setup, the simulator measures the instantaneous force exerted at each time step on the connection and simply destroys the connection when a peak of force exceeds the user specified shear strength or tensile strength.

\section{Discussion}

The proposed design shows a possibility to merge the elegant latch design from AMAS [8] and MTRAN III [18] with a higher grasping range, aiming at a higher robustness and reliability for the self-reconfiguration sequence in case of small $d_{z}$ and $d_{y y}$ misalignment between the connecting MSR units. The additionally introduced property does not come without a cost: our current prototype is more complex and not as compact as the above latching designs. The possibility to compensate for misalignment depends on the size of the latches and the size of the notches. Naturally, a compact MSR design does not leave a lot of space for both. For bigger misalignment as compensated by the latching mechanics itself a "wiggling" movement and compliance in the joints [22] can 

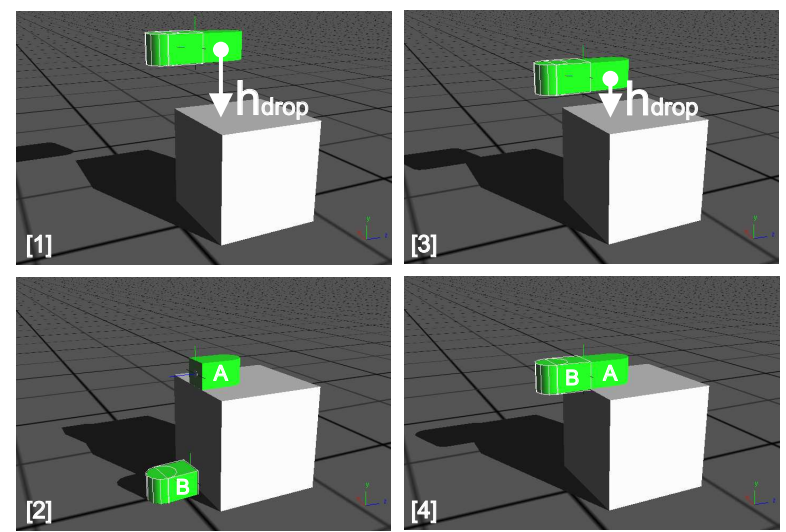

Fig. 8. Rupture experiment in simulation: dropping MSR robot units connected by an active connection mechanism. The weight of module A is largely higher than that of module B in both dropping experiments, to keep it staying on the block.

help. It is not yet known how the mechanism will perform at detaching a global MSR robot having the single units under tensile and shear stress. Hopefully tests with single connection mechanisms will enable us to gain sufficient data to feed it to the simulation environment and estimate the behavior for bigger robot structures.

\section{CONCLUSION}

We present a robust and heavy-duty active connection mechanism based on physical latching, combining the possibility to keep applying external forces inside the latches (similar designs are available with e.g. [8], [18]) with a "scooping" movement of the latch. We base our additional request for a higher $\mathrm{z}$-direction grasping range on an analysis of available active connection mechanisms for modular selfreconfigurable robots. We believe that extracted features such as genderless, hermaphrodite connectors based on our proposed latch system will support robust self-reconfiguration for our future MSR robot platform. To estimate the behavior of larger MSR robot assemblies we have developed additional features for MSR in Webots, a physics-based simulation environment. An example applying these features is shown for a simple rupture/dropping experiment in simulation. First tests with the prototype of the implemented latching mechanism demonstrate its functionality.

\section{ACKNOWLEDGMENTS}

This work was made possible thanks to financial support from Microsoft Research Cambridge, EPFL, and the Swiss National Science Foundation. We gratefully acknowledge the technical support of André Guignard, André Badertscher, Philippe Vosseler and Manuel Leitos. We would like to thank Sasha Voloshina for proof-reading.

\section{REFERENCES}

[1] S. Murata, E. Yoshida, A. Kamimura, H. Kurokawa, K. Tomita, and S. Kokaji, "M-tran: Self-reconfigurable modular robotic system," IEEE/ASME Transactions on Mechatronics, vol. 7, no. 4, pp. 431-441, December 2002.
[2] M. Yim, Y. Zhang, K. Roufas, D. Duffa, and C. Eldershaw, "Connecting and disconnecting for chain self-reconfiguration with polybot," IEEE/ASME Transactions on mechatronics, special issue on Information Technology in Mechatronics, 2003.

[3] D. Rus and M. Vona, "A physical implementation of the selfreconfigurable crystalline robot," in Proceedings of IEEE International Conference on Robotics and Automation 2000, vol. 2, 2000, pp. 17261733.

[4] S. Murata, E. Yoshida, H. Kurokawa, K. Tomita, and S. Kokaji, "Selfrepairing mechanical systems," Autonomous Robots, vol. 10, no. 1, pp. 7-21, January 2001.

[5] M. Jørgensen, E. Østergaard, and H. Lund, "Modular atron: Modules for a self-reconfigurable robot," in IEEE/RSJ Int. Conf. on Robots and Systems, Sendai, Japan, 2004, pp. 2068-2073.

[6] D. Rus, Z. Butler, K. Kotay, and M. Vona, "Self-reconfiguring robots," Communications of the ACM archive, vol. 45, pp. 39-45, March 2002.

[7] C. Ünsal, H. Kiliççöte, and P. K. Khosla, "A modular selfreconfigurable bipartite robotic system: Implementation and motion planning," Autonomous Robots, vol. 10, no. 1, pp. 23-40, January 2001.

[8] Y. Terada and S. Murata, "Modular stucture assembly using blackboard path planning systems," in International Symposium on Automation and Robotics in Construction 2006, May 2006, pp. 852-857.

[9] S. Murata, K. Kakomura, and H. Kurokawa, "Docking experiments of a modular robot by visual feedback," in IEEE/RSJ International Conference on Intelligent Robots and Systems, October 2006, pp. 625630.

[10] K. Kotay and D. Rus, "Generic distributed assembly and repair algorithms for self-reconfiguring robots," in IEEE Intl. Conf. on Intelligent Robots and Systems, Sendai, Japan, 2004.

[11] D. Daidié, O. Barbey, A. Guignard, D. Roussy, F. Guenter, A. Ijspeert, and A. Billard, "The dof-box project: An educational kit for configurable robots," in Proceedings of the 2007 IEEE/ASME International Conference on Advanced Intelligent Mechatronics (AIM2007), September 2007.

[12] F. Mondada, M. Bonani, S. Magnenat, A. Guignard, and D. Floreano, "Physical connections and cooperation in swarm robotics," in 8th Conference on Intelligent Autonomous Systems (IAS8), 2004, pp. 5360.

[13] "Molecubes for everyone." [Online]. Available: http://www.molecubes.org

[14] J. W. Suh, S. B. Homans, and M. Yim, "Telecubes: Mechanical design of a module for self-reconfigurable robotics," in IEEE Intl. Conf. on Robotics and Automation (ICRA), 2002, pp. 4095-4101.

[15] S. Goldstein, J. Campbell, and T. Mowry, "Programmable matter," Computer, vol. 38, pp. 99-101, May 2005.

[16] V. Zykov, E. Mytilinaios, B. Adams, and H. Lipson, "Self-reproducing machines," Nature, vol. 435, no. 7038, pp. 163-164, 2005.

[17] M. E. Karagozler, J. D. Campbell, G. K. Fedder, S. C. Goldstein, M. P. Weller, and B. W. Yoon, "Electrostatic latching for inter-module adhesion, power transfer, and communication in modular robots," in Proceedings of the IEEE International Conference on Intelligent Robots and Systems (IROS 07), October 2007.

[18] S. Murata and H. Kurokawa, "Self-reconfigurable robot: Shapechanging cellular robots can exceed conventional robot flexibility," IEEE Robotics \& Automation Magazine, March 2007.

[19] M. Nilsson, "Connectors for self-reconfiguring robots," IEEE/ASME Transactions on mechatronics, vol. 7, no. 4, Dezember 2002.

[20] A. Castano, A. Behar, and P. Will, "The Conro modules for reconfigurable robots," IEEE/ASME Trans. Mechatronics, vol. 7, no. 4, pp. 403-409, December 2002.

[21] V. Zykov and H. Lipson, "Fluidic stochastic modular robotics: Revisiting the system design," in Proceedings of Robotics Science and Systems Workshop on Self-Reconfigurable Modular Robots, Philadelphia PA, August 2006.

[22] M. Vona, C. Detweiler, and D. Rus, "Shady: Robust truss climbing with mechanical compliances," in International Symposium on Experimental Robotics, 2006.

[23] R. Möckel, A. Spröwitz, J. Maye, and A. J. Ijspeert, "An easy to use bluetooth scatternet protocol for fast data exchange in wireless sensor networks and autonomous robots," in IROS2007, 2007.

[24] "Webots 5, fast prototyping and simulation of mobile robots." [Online]. Available: http://www.cyberbotics.com/ 